\title{
WR+O BINARIES OBSERVED FROM THE DAO
}

\author{
G.M. HILL ${ }^{1}$ and A.B. UNDERHILL ${ }^{2}$ \\ ${ }^{1}$ Département de Physique, Université de Montréal, Canada \\ ${ }^{2}$ Department of Geophysics and Astronomy, University of British Columbia, Canada
}

\begin{abstract}
We report here the results, to date, of our monitoring of northern WR stars. New orbits have been published for the short period binaries HD 193576 and HD 214419. Of particular interest are two long period binaries with fairly eccentric orbits, HD 190918 and HD 193793. A new orbit has been determined for HD 190918. We are currently analysing spectra obtained over one cycle of the 7.9 year period of HD 193793. Other stars on our primary target list include HD 192163, HD 192641, and HD 193077.
\end{abstract}

\section{Introduction and objectives}

Since 1986 we have been using the $1.82 \mathrm{~m}$ telescope of the Dominion Astrophysical Observatory to monitor bright northern WR, Of, and $\mathrm{O}$ stars. We present here a status report on this monitoring project. A target $\mathrm{S} / \mathrm{N}$ of about 400 allows us to observe stars down to visual magnitude of about 9 or 10 (depending on conditions) for our chosen resolution of $0.45 \AA /$ pixel. The detector is a Reticon. The commissioning of a large format CCD, hopefully in the near future, will allow us to observe roughly a magnitude fainter. Most spectra are in the yellow region $(5150-6000 \AA)$ but we do have some blue and red spectra as well. On average we have about two observing runs per year with each on the order of five nights.

We are amassing a large homogenous set of WR spectra with a number of objectives in mind. Many of the published orbits of WR+O binaries were obtained with photographic spectra and have " $\mathrm{d}$ " or " $\mathrm{e}$ " ratings in Batten's catalogue. High S/N digital spectra should allow us to obtain improved orbits for these stars. There are a number of WR stars, for which conflicting claims of binarity can be found in the literature. In some cases, stars are almost certainly binaries but orbits have never been published. We are monitoring some of these stars to investigate their status and obtain orbits where possible. Some WR stars are assumed to be single. Confirmation of this is useful and we are obtaining spectra of a few such stars.

A large bank of homogenous spectra is crucial to the study of line profile variations in both single and binary stars. These variations may result from such phenomena as blobs in stellar winds, filaments in a disk, colliding winds, nonradial pulsation in stellar photospheres etc. With a data set consisting of spectra at a variety of resolutions and $\mathrm{S} / \mathrm{N}$ ratios, it is harder to properly study these phenomena which yield important information about the nature of WR stars and the physics of their line-emitting regions.

Currently our primary targets include HD 190918, HD 193793, HD 193077, HD 192641, and HD 192163. HD 190918 is discussed below. We have about two dozen spectra of HD 193793 (WC7+O4-5) obtained over one cycle of 
its 7.9 year period. HD 193077 is classified as WN5+abs and various claims exist for binary periods of a few days ranging up to a few years. HD 192641 is a very interesting $\mathrm{WC} 7+$ abs object that may be a binary with a period on the order of a decade. HD 192163 is probably a single WN6 star but merits some study given the occasional claim about variability.

\section{Recent results}

Early emphasis was on HD 193576 (V444 Cyg) and HD 214419 (CQ Cep) and new orbits have been published for these short period binaries (Underhill et al. 1988a,1988b, 1990a). Papers have also been published on HD 191765 (Underhill et al. 1990b) and HD 192641 (Underhill 1992). Recently most of our attention has been on the long period binary HD 190918 (WN4.5+09.5Ia).

A new orbit has been determined for HD 190918 using the He II $\lambda 5412$, $\mathrm{O}$ III $\lambda 5592$, and He I $\lambda 5876$ absorption lines and the He II $\lambda 5412$ emission line. We find $P=112.4 \pm 0.2 \mathrm{~d}$ and $e=0.39 \pm 0.07$. For the $O$ star we find $\omega=198^{\circ} .9 \pm 10.7, T_{\text {peri }}=2447420.5 \pm 3.6, \gamma=-20.9 \pm 0.7 \mathrm{~km} \mathrm{~s}^{-1}$, and $K=16.9 \pm 2.1 \mathrm{~km} \mathrm{~s}^{-1}$. For the WR star, $\gamma=70.2 \pm 4.6 \mathrm{~km} \mathrm{~s}^{-1}$, and $K=34.4 \pm 7.4 \mathrm{~km} \mathrm{~s}^{-1}$. Fig. 1 presents plots of our orbital solution together with the measured velocities. Given the late $O$ spectral type of the primary, the orbital inclination is about 20 to 25 degrees. For this range of $i$ the 0 star has a mass in the range 10 to $21 \mathrm{M}_{\odot}$ and the WR star has a mass in the range 5 to $10 \mathrm{M}_{\odot}$. If the 0 star is truly a supergiant, then the larger values are more appropriate.

HD 190918 is interesting for a number of reasons. It is one of a small number of SB2 systems. It is relatively bright, yet correct orbital elements have never been published. A valuable aspect of HD 190918 is demonstrated by figure 7 of Abbott et al. (1986). The WR component has relatively low values of $M, \dot{M}$, and $\mathrm{v}_{\infty}$. Any study of how various physical quantities depend on any of these parameters will be sensitive to the extremum points so it is important to determine these quantities as accurately as possible.

Perhaps, with respect to the topic of this meeting, the most interesting aspect of HD 190918 is the possiblility that the effects of wind-wind collisions will be observable for this system. At periastron, the two stars are separated by only about $200 \mathrm{R}_{\odot}$ and both stars have substantial winds. The fairly eccentric orbit means the 0 star "samples" the WR wind at a range of distances. Theoretical studies imply that systems such as $\gamma$ Vel and HD 190918 should be rewarding examples of colliding wind binaries. The orbital elements of HD 190918 are somewhat similar to those of $\gamma$ Vel, for which phase-dependant spectral changes have been interpreted as arising from colliding winds (St-Louis et al. 1993). X-ray observations are consistent with the colliding wind scenario as well (Willis et al. 1993)

Unfortunately, to our knowledge, HD 190918 has not been studied in X- 

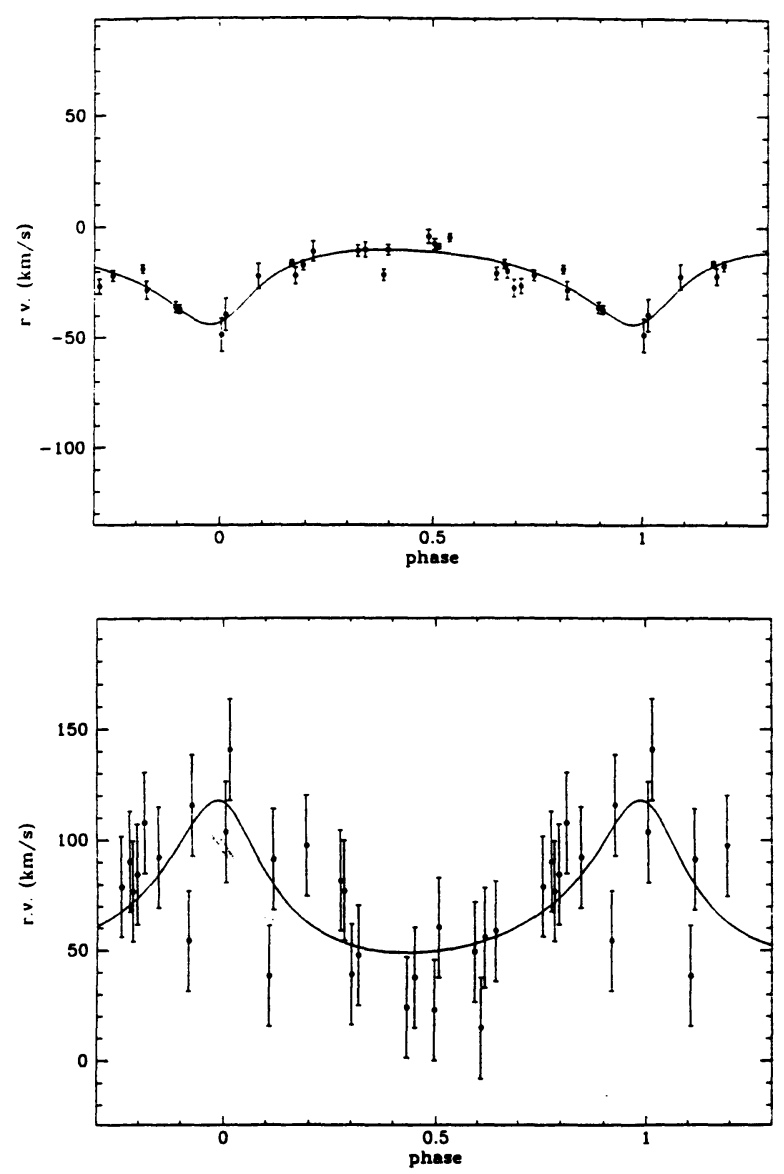

Fig. 1. Fig. 1. HD 190918: The radial velocity curve shown by the $O$ star (upper) and WR star (lower)

rays. Although we have enough spectra to determine an orbit, we do not have extensive, multiple cycle coverage of periastron to properly study line profile variations. Despite these difficulties, the spectra of HD 190918 do imply that wind collision effects might be present. The narrow $\mathrm{C}$ III $\lambda 5696$ emission line originating from a region near the 0 star is subtly variable. It is not obvious that the variations are phase-locked though. More interesting is the appearance of two other lines. Fig. 2 shows two of our spectra. The top spectrum was obtained about 0.3 cycles before periastron and below it is a spectrum taken near periastron. Note the difference in the N IV $\lambda 5203$ profiles. Also to be noted is the extra absorption component (at periastron) just shortward of He I $\lambda 5876$. We plan to continue observing HD 190918 in an attempt to quantify these variations and further investigate the possiblity of colliding winds in this important binary. We also urge X-ray astronomers 


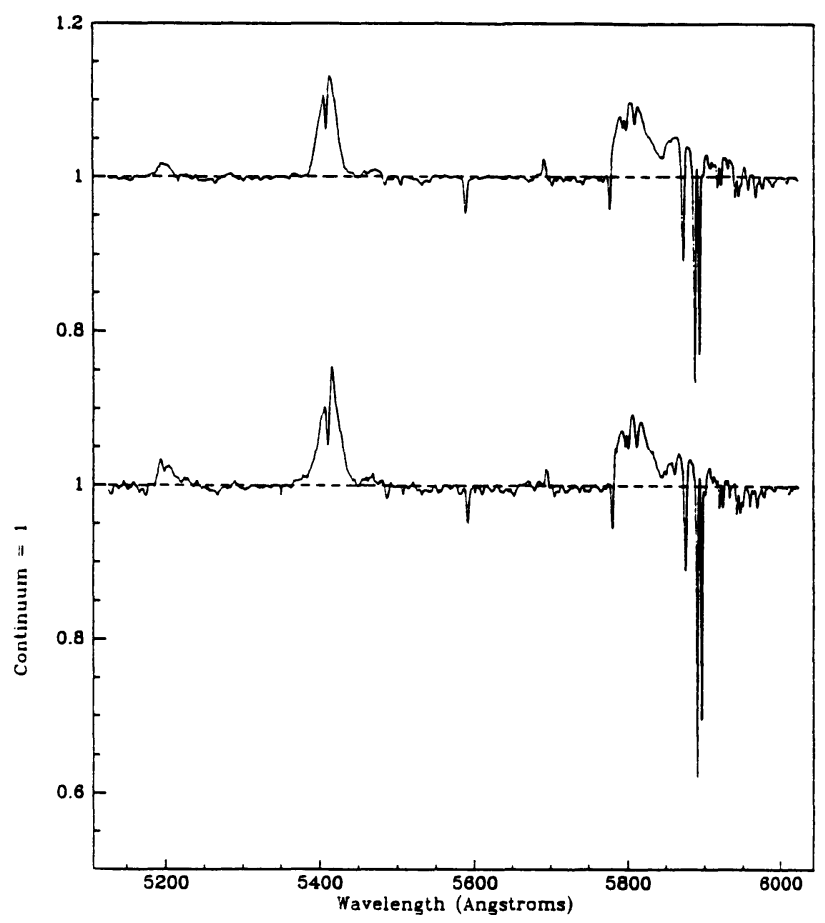

Fig. 2. Fig. 2. Spectra of HD 190918 taken at phase 0.7 (top) and 0.0 (bottom)

to consider adding HD 190918 to their target list.

\section{References}

Abbott, D.C, Bieging, J.H., Churchwell, E.B., Torres, A.V. 1986, ApJ 303, 239

St-Louis, N., Willis, A.J., Stevens, I.R. 1993, ApJ 415, 298

Underhill, A.B., Yang, S., Hill, G.M. 1988, PASP 100, 741

Underhill, A.B., Yang, S., Hill, G.M. 1988, PASP 100, 1256

Underhill, A.B., Gilroy, K.K., Hill, G.M. 1990a, $A p J$ 351, 651

Underhill, A.B., Gilroy, K.K., Hill, G.M., Dinshaw N. 1990b, ApJ 351, 666

Underhill, A.B. 1992, ApJ 398, 636

Willis, A.J. et al. 1994, in: Proc. Me aux Coudres Workshop, in press

\section{DISCUSSION:}

Cohen: I would urge you to seek $5165 \mathrm{~A} \mathrm{C}_{2}$ Swan bands in your yellow spectra. The route to making gains from neutral species such as CI must pass through molecules such as $\mathrm{C}_{2}$ and $\mathrm{C}_{3}$. Your spectra would reveal $\mathrm{C}_{2}$, if visible. You might look in your time resolved spectra of $\mathrm{HD}$ 193793 and in any WC9 stars with known well-developed dust shells. $\mathrm{C}_{2}$ could be in emission or absorption.

Hill: I will check our spectra. 